\title{
Fast Tracking for the Second Level Trigger of the ATLAS Experiment Using Silicon Detectors Data
}

\author{
C. Schiavi, M. Cervetto, F. Parodi, Dipartimento di Fisica e INFN, Genova, Italy \\ N. Kostantinidis, M. Sutton, University College London, London, UK \\ J. Baines, D. Emeliyanov, Rutherford Appleton Laboratory, Chilton, Didcot, UK \\ H. Drevermann, CERN, Geneva, Swirtzerland
}

\begin{abstract}
Online track reconstruction is an important ingredient for event selection at Large Hadron Collider (LHC) experiments. In the ATLAS experiment the first stage where this goal will be achievable is the software-based Second Level Trigger (LVL2). In this contribution we present an algorithm for fast pattern recognition and reconstruction of charged tracks and of the primary vertex in the framework of the High Level Trigger (HLT) of ATLAS. The pattern recognition makes extensive use of Monte Carlo Look Up Tables to quickly identify, in the innermost layers of the ATLAS silicon detectors, triplets of space points reconstructed from hits produced by the same track. The reconstruction strategy is compared, in the ATLAS LVL2 framework, with an alternative tracking algorithm, showing the complementarity of the two approaches. The algorithm's performance is presented for different event topologies and luminosities, showing good tracking capabilities and uniform results with mean execution times which are compatible with the LVL2 requirements.
\end{abstract}

Index Terms-ATLAS, Trigger, Tracking

\section{INTRODUCTION}

The ATLAS detector will operate at the LHC collider studying $p p$ collisions at $14 \mathrm{TeV}$ center of mass energy. The region around the $p p$ interaction point will be characterized by a very high density of charged tracks, which can be reconstructed only by using high granularity detectors granting low occupancy. The algorithm presented here aims at charged track reconstruction exploiting the precise 3D measurements of the Pixel detector (the innermost part of the ATLAS tracking system) within the time constraint of the Second Level trigger (on average, a decision has to be taken every $10 \mathrm{~ms}$ ).

\section{ATLAS}

The ATLAS (A Toroidal LHC ApparatuS) experiment will start taking data in April 2007 at the Large Hadron Collider (LHC), a $p p$ collider, currently under construction by the European Organization for Nuclear Research (CERN), characterized by a $14 \mathrm{TeV}$ center of mass energy and a design luminosity of $10^{34} \mathrm{~cm}^{-2} \mathrm{~s}^{-1}$.

ATLAS has been designed as a multipurpose experiment, to be capable both of detecting and measuring new physical phenomena predicted by currently available theories, like evidence for Higgs bosons or supersymmetrical particles, and of performing precision Standard Model (SM) measurements; at the same time it must be also open to unexpected signals from unpredicted physics scenarios and has thus to be sensitive to any kind of event topology.

To achieve this goal, the ATLAS detector is equipped, moving from the inside out, with tracking and particle identification detectors (Pixel, SCT silicon strips and TRT straw tubes) forming the so called Inner Detector (ID), Liquid Argon (LAr) electromagnetic and Tile hadronic calorimeters, and the outer muon system, designed both for tracking (Monitored Drift Tubes and Cathode Strip Chambers) and trigger purposes (Resistive Plate and Thin Gap Chambers).

The innermost tracking detector (the Pixel detector) is composed by a central barrel region and two end-caps, one on each side of the barrel region, increasing the angular coverage; the barrel is made by three cylindrical layers with different radii, $R=5.05,8.85,12.25 \mathrm{~cm}$, whose axis corresponds to the beam line; each end-cap is instead made of three disc layers, placed at different positions along the beam line. All detector layers are based on the same basic element, the Pixel module, containing a matrix of $320 \times 144$ Silicon Pixel sensors; the pitch of the sensors is $50 \mu \mathrm{m}$ in the transverse direction and $400 \mu \mathrm{m}$ along the beam axis in the barrel region and along the radial direction in the end-caps. For the initial period of the data taking a reduced ID layout (lacking the intermediate Pixel layers and the TRT $\mathrm{C}$ wheels at $|\eta|>1.7$ ) has been approved for budget reasons.

Operation at LHC means coping, at design luminosity, with $\sim 23 \mathrm{pp}$ interactions every $25 \mathrm{~ns}$; this very high rate obviously poses stringent design demands on both the detectors and the Trigger and Data Acquisition (TDAQ) systems.

From the hardware side this means, as an example, that every subdetector must be equipped with built-in pipeline memories to temporarily store events while the first level trigger decision is taken; furthermore the entire detector, which is more or less $20 \mathrm{~m}$ tall, must be synchronized to better than $25 \mathrm{~ns}$ in order to perform a correct event building.

On the other side the high number of detector channels leads to a mean event size of $\sim 1.5 \mathrm{MB}$; from the TDAQ point of view, this both means facing a very challenging networking task and limiting the final event storage rate to a maximum value of $\sim 200 \mathrm{~Hz}$. This last requirement must be fulfilled through the event rejection performed by the trigger system. 


\section{THE ATLAS TRIGGER SYSTEM}

In the ATLAS experiment, reduction of the $1 \mathrm{GHz}$ interaction rate down to the $200 \mathrm{~Hz}$ maximum event data storage rate is provided through three different trigger selection layers.

- The hardware-based First Level trigger (LVL1) performs a preliminary rejection using only reduced granularity data coming from calorimeters and muon detectors, within a $2 \mu$ s fixed latency, producing an average output rate of $75 \mathrm{kHz}$. Further event selection is then performed by software tools running on dedicated commercial processor farms and is divided in two layers, Second Level trigger (LVL2) and Event Filter (EF), collectively referenced as HLT [1].

- Reconstruction at LVL2, seeded by information collected at LVL1, can exploit full granularity information from all ATLAS subdetectors, processing in parallel data contained inside one or more geometrical regions identified at LVL1, the so called Regions of Interest (RoI). Event selection is designed in order to provide an output rate below $2 \mathrm{kHz}$, and must be performed with a mean processing time of $10 \mathrm{~ms}$ per event; obviously the limited execution time greatly constraints the LVL2 reconstruction algorithms, which have to be kept as simple as possible and have to be optimized for timing performance.

- The EF selection, which can be in its turn seeded by results obtained at LVL2, has much looser time constraints, with a $2 \mathrm{~s}$ mean execution time, and can thus use reconstruction algorithms with potential access to the entire event data and which are much more similar to the tools used for offline analysis.

\section{A. Fast Tracking Algorithm for the Second Level Trigger}

At LVL2, Inner Detector data is available for track reconstruction. Fast pattern recognition can be achieved by searching for the innermost triplet among the hits produced by each track. The spatial coordinates (space points) of the hits of each triplet allow to compute, using a simple helix parametrization, the track parameters with good resolution (especially for the impact parameters with respect to the primary interaction vertex).

Since at the LHC design (high) luminosity on every bunch crossing about 25 events are spread along the beam direction by $\sigma(z)=5.6 \mathrm{~cm}$, one of the most important tasks for the tracking is the determination of the $z$ coordinate of the primary vertex of the main interesting event (characterized by large transverse energy) allowing rejection of background hits from other soft interaction vertices.

The design of the algorithm presented in this paper is highly modular, so it can be easily decomposed in terms of its component blocks. After having explained the principle used to group physical detector modules into logical layers with the aid of Monte Carlo maps, a detailed description of the main algorithm blocks will be given.

1) Logical layers and Monte Carlo learning: A logical layer can be roughly defined as a set of detector modules from different physical layers playing the same role during track reconstruction.

Logical layers are built examining Monte Carlo tracks that at least produced a space point on the B-layer, ordering and numbering their hits with increasing $r$ values and finally grouping together the modules containing space points with the same number. So, as an example, the second logical layer contains all the modules on which the second space point from at least one Monte Carlo track lays.

Obviously this definition implies that the first logical layer corresponds exactly to the the first Pixel layer (the so called Blayer); this choice follows from the fact that the space points it provides are very close to the interaction region and thus mainly contribute to an accurate evaluation of track impact parameter.

2) Space point sorting: The first algorithmic operation is sorting the space points retrieved from the LVL1 RoI putting them in a map according to their physical module address in order to speed-up the following data access.

3) Track seeds formation: Using the sorted map and a LookUp Table (LUT) linking each module within the B-layer to the ones belonging to second logical layer track seeds are formed by two space points and interpolated with a straight line.

The line defined by each seed is extrapolated back to the beam line and the transverse and longitudinal impact parameter are computed.

The space point pair is accepted if it has a small impact parameter with the primary vertex in the transverse plane (coincident, with good approximation, at LHC with the origin in the transverse plane). The requirement on the value of the transverse impact parameter can be actually used to tune the lowest $p_{T}$ threshold for the track reconstruction.

4) Primary vertex reconstruction: The $\mathrm{z}$ coordinate of the primary vertex can be computed in the first stage of the processing by histogramming the $z$ impact parameter for all the accepted seeds. The coordinate of the maximum of the histogram is taken as an estimate of $z_{\text {vertex }}$.

More than one candidate is retained to improve efficiency ( $\epsilon \sim 85-95 \%$ depending on the luminosity and event topology). Efficient primary vertex reconstruction is needed to guarantee uniform performance in the different luminosity regimes.

5) Track extension: Each track seed is extended adding a third space point. The extrapolation is performed using a MC map giving, for each seed, a set of module lists where further hits may lay (road); a subset of module is selected for each road according to their distance with respect to the primary vertex. Space points from the selected modules are used to extend the seed if they are compatible with its linear extrapolation.

The selected triplets of space points can have space points in common. It is then necessary to remove the assignment ambiguities and terminate the processing with an unambiguous use of the space points.

The procedure for the removal of the overlaps consists in grouping the tracks sharing at least one space-point; this grouping is performed following an associative rule, so that two tracks in the same group can be non overlapping if both share a cluster with a third track. 


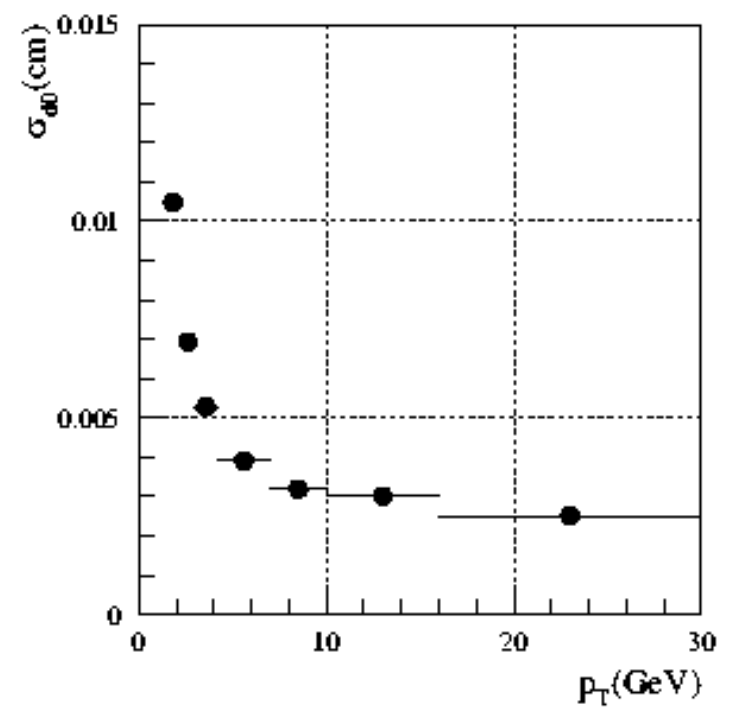

Fig. 1. Transverse impact parameter $\left(d_{0}\right)$ resolution as a function of $p_{T}$.

The tracks in a group are then ordered according to the distance to the seed extrapolation. The track with the smallest distance is retained, those sharing a space-point with the first are discarded and the others are regrouped and the procedure is iterated.

The spacepoints triplets are then fitted (with a circle in the $r-\phi$ plane, with a line in the $r-z$ plane) and identified as candidate tracks.

\section{B. Performance}

The algorithm reconstructs tracks in jets with an efficiency ranging from $80 \%$ to $90 \%$ (depending on the luminosity and event topology). Single electrons are reconstructed with an efficiency of about $95 \%$ for all the luminosities.

The track resolutions are summarized in Table I.

\begin{tabular}{|l|l|}
\hline$\sigma\left(1 / p_{T}\right)\left(\mathrm{GeV}^{-1}\right)$ & 0.006 \\
\hline$\sigma(\phi)(\mathrm{rad})$ & $0.7 \times 10^{-3}$ \\
\hline$\sigma(\eta)$ & $2.2 \times 10^{-2}$ \\
\hline$\sigma\left(z_{0}\right)(\mu \mathrm{m})$ & 340 \\
\hline
\end{tabular}

TABLE I

Track parameters resolution for $\left(p_{T}>1\right)$.

The transverse impact parameter $\left(d_{0}\right)$ resolution is shown in Figure 1 as a function of $p_{T}$, the asymptotic value for the $d_{0}$ resolution turns out to be $30 \mu \mathrm{m}$.

The results of timing measurements on a $2.4 \mathrm{GHz} \mathrm{PC}$ processing RoIs from isolated electrons and $b$-jets (signal only) are given in Table II.

The total algorithmic time increases, in the worst case, roughly by a factor $\sim 1.4 \mathrm{~ms}$ at initial luminosity and a by factor $\sim 2.5 \mathrm{~ms}$ at design luminosity. The timing performance is well within the LVL2 constraints.

\begin{tabular}{|l|c|c|c|c|}
\hline \hline & Sorting & Seeding & Extension & Total \\
\hline b-jets & $\sim 0.2 \mathrm{~ms}$ & $\sim 0.5 \mathrm{~ms}$ & $\sim 0.3 \mathrm{~ms}$ & $\sim \mathbf{1 ~} \mathbf{~ s}$ \\
\hline isolated e & $\sim 0.02 \mathrm{~ms}$ & $\sim 0.1 \mathrm{~ms}$ & $\sim 0.1 \mathrm{~ms}$ & $\sim \mathbf{0 . 2 2} \mathbf{~ m s}$ \\
\hline \multicolumn{5}{c}{ TABLE II } \\
Timing measurements for the different steps of the algorithm.
\end{tabular}

\section{APPLICATION: ONLINE $b$-TAGGING SELECTION}

Given the good transverse impact parameter resolution, a natural application of the fast tracking algorithm described above is $b$-jet selection at LVL2.

The use of $b$-jet tagging selection at LVL2/EF could improve the flexibility of the HLT scheme and possibly extend its physics performance. In particular, for topologies containing multi $b$-jets, the ability to separate $b$-jets from light quark and gluon jets could increase the acceptance for signal events (if the use of lower jet $E_{T}$ thresholds at LVL1 is feasible) or reduce the background (and hence the rate) for events containing $b$-jets that have already been selected by other triggers.

\section{A. Samples used for b-tagging studies}

The performance of the $b$-tagging selection has been studied using $b$-jets from Higgs boson (120 $\mathrm{GeV}$ mass) decay as the signal sample. A representative background sample was created by artificially replacing the $b$-jets with $u$-jets in the Higgs decay. The LVL1 RoI was simulated by a region $\Delta \phi \times \Delta \eta=0.4 \times 0.4$ centered on the quark and anti-quark directions.

\section{B. The b-tagging algorithm}

The $b$-jet selection is performed using the transverse impact parameter of its tracks. For each reconstructed track the significance of the transverse impact parameter $S=d_{0} / s\left(d_{0}\right)$ is computed; the error on the impact parameter $s\left(d_{0}\right)$ is parametrized, using simulated events, as a function of $p_{T}$.

The $b$-jet estimator is then built using the likelihood-ratio method: for each track $(i)$, the ratio of the probability densities for the track to come from a $b$-jet or a $u$-jet is calculated: $f_{b}\left(S_{i}\right) / f_{u}\left(S_{i}\right)$; the product $W$ of these ratios over all reconstructed tracks in the jet is computed and the final tagging variable $X=W /(1+W)$ is defined. Jets are tagged as $b$-jets if $X \sim 1$ and $u$-jets if $X \sim 0$. The selection efficiency of the $b$-jets and the rejection of light flavour jets can be tuned by cutting on the $X$ variable.

Figure 2 shows the distributions of the discriminant variable $X$ for $b$ jets and $u$ jets.

\section{C. b-jet tagging performance}

The $b$-tagging algorithm has been characterized on single $b$ jets. The efficiencies for $b$-jets $\left(e_{b}\right)$ and rejection factors $\left(R_{u}\right)$ against $u$-jets (defined as the inverse of the efficiency for $u$-jets) are given in Table 3 .

The performance is robust with respect to luminosity and event topology. The rejection, although modest, is still useful to 


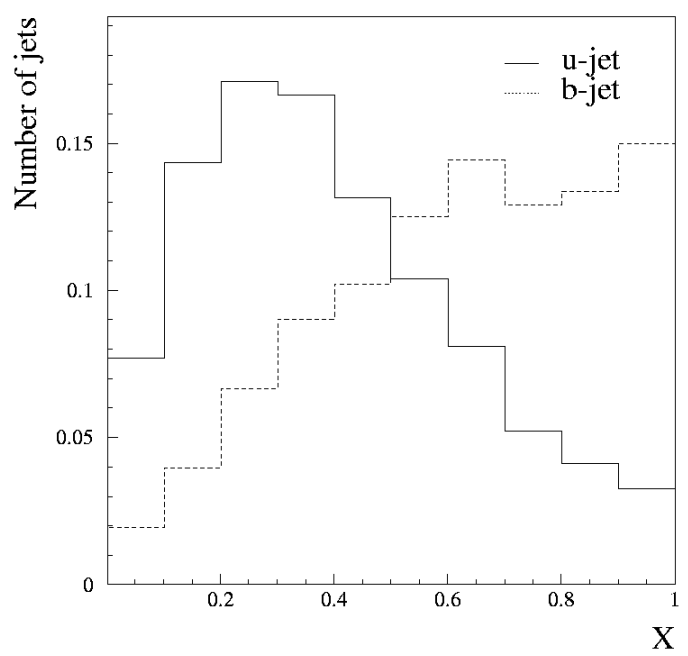

Fig. 2. Distribution of discriminant variable $X$ for $b$ jets (full line) and $u$ jets (dashed line).

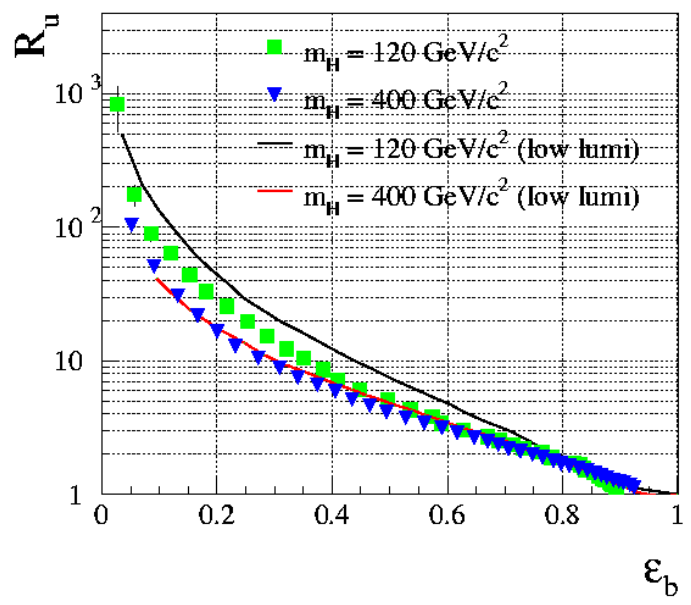

Fig. 3. Rejection for light jets as a function of the efficiency for b-jets for different luminosities and event topologies.

increase the acceptance of multi $b$-jets events (SUSY channels) and, more generally, to increase the flexibility of the trigger scheme.

\section{APPLiCATION: ONLINE ELECTRON AND PHOTON SELECTION}

Another interesting application of the algorithm here described is the operation in the isolated electron and photon triggers, demonstrating the flexibility of this approach to the LVL2 track reconstruction problem. Example applications of these trigger menus are the selection of Higgs boson decay channels like $H \rightarrow 4 e$ and $H \rightarrow \gamma \gamma$, whose combination covers the interesting mass range $100 \mathrm{GeV}<m_{H}<150 \mathrm{GeV}$ [3]. Since the event topology is quite far from the one faced for the $b$-tagging application, the configuration and the tuning of the algorithm also follow different strategies.

\section{A. Algorithm tuning for isolated electron reconstruction}

First of all the main difference is that, given the low charged track multiplicity of this kind of events (in most cases just one high $p_{T}$ charged track is contained in each RoI), no primary vertex reconstruction is possible; this means that no cut is applied on the $z_{0}$ impact parameter of the reconstructed track seeds, apart from the very weak request of compatibility with the average primary vertex spread.

On the other side, since the electrons tagged at LVL1 are characterized by high $p_{T}$ values (exceeding 25 to $30 \mathrm{GeV}$, depending on the luminosity conditions) and come from the decay of short lived particles, much tighter cuts are applied on the transverse impact parameter, both in the seeding and in the extension phases. This obviously reduces the $p_{T}$ acceptance and also has a big impact on timing performance, which results greatly improved with respect to the one obtained for $b$-jet reconstruction.

\section{B. LVL2 tracking and the electron and photon selection}

Tracks reconstructed at LVL2 are used in the isolated electron menu to confirm candidates identified by clusters in the EM LAr calorimeter; this is achieved using geometrical $(\eta, \phi)$ matching with the position of the cluster. Furthermore, the information on the reconstructed transverse momentum is combined with the energy measurement in the calorimeter, evaluating the $E_{T} / p_{T}$ ratio in order to perform $e / \pi$ separation. Results obtained from studies on simulated single isolated electron samples show that the fake electron candidates rejection obtained employing the LVL2 ID tracking information is significant and, in particular, is crucial in order to meet the rate constraints at the boundary between the LVL2 and the EF processing.

In the case of the isolated photon trigger, information from tracking reconstruction is instead used to apply a track veto from the ID on calorimeter clusters identified as photon candidates.

\section{FAST TRACKING: AN ALTERNATIVE APPROACH}

An alternative approach [4] for track reconstruction based on histogramming technique has been developed in ATLAS, it is composed of several sub-algorithm.

- ZFinder Hits are selected in narrow $\phi$ slices, pairs of hits in each slice are extrapolated back to the beam line entering the $z$ of the intersection in a histogram. The $z$ value corresponding to the peak of the histogram is taken as that of the primary vertex.

- HitFilter Puts all hits into a histogram binned in $\phi$ and $\eta$. It finds clusters of hits within the histogram and creates group of hits if the cluster contains contributions from more than a given number of layers. 
- GroupCleaner Splits hits groups into tracks and removes noise hits from group. Each triplet of hits forms a potential track, groups of triplets with similar parameters are formed. Track candidates are accepted if a groups contains enough hits.

- TrackFitter Verifies track candidates and finds track parameters by using a fast Object Oriented implementation of the Kalman filter algorithm.

This approach has given similar results with the one described above, both in terms of physics and timing performance, and is in many ways complementary since it has different stregths and weakness.

In particular it has been designed to use all the the hits from the silicon detectors and hence to determine precisely the track directions $(\phi, \eta)$ and the transverse momentum $\left(p_{T}\right)$; it also allows a more flexible use of different detector configurations. On the other hand since the track search doesn't start from the inside to the outside of the detector the resolution on impact parameters is slightly less enhanced and the lack of an ambiguity solver potentially lead to larger combinatorics contribution.

\section{CONCLUSION}

The pattern recognition algorithm for the ATLAS second level trigger presented here is based on the reconstruction of triplets of silicon hits identifying the innermost segment of the charged tracks. The algorithm's performance shows good tracking capabilities and uniform results within execution times largely compatible with the LVL2 requirements.

The algorithm can used as a standalone algorithm or as a first stage of more complex pattern recognition. As a standalone algorithm it has been extensively used both for $b$-tagging studies and reconstruction of isolated electrons.

The complementary approaches to track reconstruction provide greater flexibility by allowing the optimum reconstruction tools to be used for specific trigger selections. This, together with the ability to cross-check performance, is crucial in order to maximize performance in the challenging environment of the second level trigger

\section{REFERENCES}

[1] ATLAS HLT/DAQ/DCS Group, ATLAS High-Level Trigger, Data Acquisition and Controls Technical Design Report, CERN/LHCC/2003-022 ATLAS-TDR-016 (2003).

[2] M. Cervetto, F. Parodi, P. Morettini and C. Schiavi, ATL-COM-DAQ-200025 (2003)

[3] V. Perez-Reale et al, Triggering Standard Model Higgs processes in the ATLAS experiment, Proceedings of "Physics at LHC" 2004 conference, Vienna 13-17th of July 2004, Czechoslovak Journal of Physics, Vol. 54 (2004), Suppl. A.

[4] N. Konstantinidis, H.Drevermann, ATL-COM-DAQ-2003-040 (2003) 